\title{
Interpretation of Coagulation Tendency Contributing to Thrombosis in Vector-Borne Diseases (Ehrlichiosis, Anaplasmosis, Leishmaniosis, and Dirofilariasis) among Dogs
}

\author{
Serdar Pasa, Kerem Ural \& Mehmet Gultekin
}

\begin{abstract}
Background: Vector-borne infectious and zoonotic diseases are an important health problem that directly affects human and animal health negatively. Results through evaluation of coagulation disorders among vector-borne diseases should be of beneficial for both human and dogs studies. According to the present author's knowledge reports regarding changes in platelet (PLT) count, prothrombin time (PT), activated partial thromboplastin time (APTT), fibrinogen (FIB) and D-dimer levels in dogs naturally infected with one or more vector-borne pathogens are lacking. Therefore, the present study was aimed to detecting those parameters for relation between diagnosis and prognosis of vector-borne diseases among dogs.

Materials, Methods \& Results: The material of this study was $46 \mathrm{dogs}$ (36 were naturally infected with vector-borne diseases and 10 were healthy) from different breed, age and of both sexes. Venous blood samples were obtained to detect PLT counts, antibodies of ehrlichiosis, anaplasmosis, borreliosis, leishmaniosis and antigens of Dirofilaria immitis. The diagnosis of vector-borne diseases was performed by using a commercial ELISA assay kits. PLT count was performed with an automated blood cell counter analyser. In addition, PT, APTT and FIB concentrations were measured using a microcoagulometer. D-dimer concentrations were determined using fluorescence immunoassay rapid quantitave test analyser. Subgroups were formed according to the number of cases and the distribution of vector-borne agent. Statistically significant decreased PLT count was found in dogs mono infected with ehrlichiosis compared to healthy dogs $(P<$ 0.001). Changes in mean PT value in the studied animals did not show statistically significant differences among the groups $(P>0.05)$. APTT values in the ehrlichiosis mono infection group were significantly higher than that of the healthy control $(P<0.01)$. A significant increase in FIB levels were detected for ehrlichiosis mono infection and ehrlichiosis - leishmaniosis co infection versus healthy control $(P<0.001)$. Plasma D-dimer concentrations were found to be higher in all groups infected with vector borne diseases compared to healthy group $(P<0.001)$ and the differences between infected groups were not statistically significant.

Discussion: Bleeding disorders such as epistaxis, haematuria and haemorrhagic diarrhoea has been reported in dogs with vector-borne diseases. These disorders represent the main cause of death in dogs. In the present study, thrombocytopenia was observed in dogs mono infected with ehrlichiosis compared to healthy. This finding is in agreement with those reported in dogs with ehrlichiosis. Plasma FIB is one of the most important factors in the coagulation cascade. In the present study, a significant difference between dogs with ehrlichiosis mono infection and ehrlichiosis - leishmaniosis co infection versus healthy controls group was observed. PT and APTT are commonly used in evaluating dogs with bleeding tendencies. In the present study, a significant difference between dogs with ehrlichiosis and with healthy control was observed in APTT values, however, differences in PT values compared to healthy dogs were insignificant. No statistical difference in PT values might be related to the lower sensitivity of the commercial PT assays. In dogs, D-dimer concentrations can be elevated due to disseminated intravascular coagulopathy, infections, metabolic disorders, neoplasia and post-surgically. In the present study, a significant increase in D-dimer concentration was observed in all dogs with vector-borne diseases. This finding points to the activation of the fibrinolysis system in consequence of thrombophilia. In conclusion, elevations presented in coagulation biomarkers such as APTT, FIB and D-dimer in the present study were interpreted as with the effects of vector-borne diseases. It may be briefly suggested that D-dimer levels as a marker of pro-coagulatory activity, as well as fibrinolysis, indicates the highly active and excessive coagulation, and all through are risk factors for thromboembolic disorders. Therefore, these findings should be considered in the diagnosis, treatment and prognosis of the vector-borne diseases in dogs.
\end{abstract}

Keywords: vector-borne, disease, dog, D-dimer, coagulation, thrombocytopenia 


\section{INTRODUCTION}

Infectious and zoonotic vector-borne diseases possesses significant health problem directly affecting human and animal health. Vectors such as ticks and flies play an important role in the transport of many rickettsial, parasitic, spirochetal and bacterial diseases. Diseases transmitted via ticks/flies accompany serious infection, organ dysfunction and death. Vector-borne diseases such as leishmaniosis, ehrlichiosis, borreliosis, and dirofilariasis are very important because of increased importation of different dog breeds from foreign countries, as well as control requirements of exposed pets [23,25,26,28].

Vector-borne diseases represent a diagnostic challenge as they usually cause non-specific signs and clinical manifestations which may vary according to the pathogen involved, parasitemia or bacteraemia level, immune status and age of the dog [1,26,31]. In veterinary medical practice, sometimes dogs are presented for episodes of bleeding tendencies for unknown reason. In evaluating dogs with bleeding tendencies, coagulation tests are very important. These tests include platelet (PLT) count and buccal mucosal bleed time for primary haemostasis; one-stage prothrombin time (PT), activated partial thromboplastin time (APTT), and activated clotting time (ACT) for secondary haemostasis; products such as fibrinogen (FIB) degradation products and D-dimers for fibrinolysis; and measurement of proteins $\mathrm{C}$ and $\mathrm{S}$ and antithrombin activity to evaluate endogenous anticoagulant potential $[6,35]$.

Vector-borne diseases are mostly characterized by fever and other relevant signs and infection might exist occur not only in dogs, but also in people. It is clear that a literature-based search on zoonotic ailments necessitated a clinically-based study of the increasingly prevalent ehrlichiosis, anaplasmosis and leishmaniosis. In Turkey apart from some limited case reports or small population studies, no clinical based and detailed study is evident. Thus the lack of studies and ethiopathological analysis, a detailed survey is promptly required.

Results through evaluation of coagulation disorders among vector borne diseases should be of beneficial for both human and dogs studies. According to the present authors knowledge reports regarding coagulation and haemostatic profile alterations and related studies composed of changes in APTT, PT, FIB and D-dimer levels in dogs naturally infected with one or more vector-borne pathogens are lacking. Therefore, the present study was aimed to detecting those parameters for relation between diagnosis and prognosis of vector-borne diseases among dogs.

\section{MATERIALS AND METHODS}

\section{Material, sample collection and diagnosis}

The material of this study was 46 dogs (36 were naturally infected with vector-borne diseases and 10 were healthy) from different breed, age and of both sexes. All of the animals referred from the around the Aegean coast of Turkey. The data collector recorded a detailed anamnesis and, performed a full physical examination and findings were recorded for each dog. Clinical signs of the infected dogs were consistent with vector-borne diseases based on weight loss, pale mucous membranes and lymphadenopathy. Venous blood samples (EDTA, citrate and serum gel tubes) were obtained from the cephalic veins of dogs to detect PLT counts, antibodies of ehrlichiosis, anaplasmosis, borreliosis, leishmaniosis and antigens of Dirofilaria immitis. The diagnosis of vector-borne diseases was performed by using a commercial ELISA assay kits (Snap 4DX plus and Snap Leish test) ${ }^{1}$. In addition, differential diagnosis of some other infectious diseases (babesiosis, hepatozoonosis) which could cause similar clinical or laboratory findings was searched by cytological examination of blood samples. Healthy dogs as control group were selected according to anamnesis, physical examination, hematological and biochemical findings.

\section{Determination of coagulation profile}

PLT count was performed with an automated blood cell counter analyser Abacus Junior Vet $5^{2}$. In addition, PT, APTT and FIB concentrations were measured using a semi-automated four channel microcoagulometer ${ }^{3}$. D-dimer concentrations were determined using Finecare fluorescence immunoassay rapid quantitave test analyser ${ }^{4}$. The dogs diagnosed naturally infected with vector-borne diseases were classified into two groups as mono or co-infected. Subgroups were formed according to the number of cases and the distribution of infectious agent.

\section{Statistical analysis}

Statistical analysis of the results was performed by use of software package program ${ }^{5}$. In each group arithmetic mean $(\mathrm{X})$ and standard deviation $(\mathrm{SD})$ values were calculated. Distribution of the values was evalu- 
ated by Kolmogorov-Smirnov or Shapiro-Wilk test. Parameters not normally distributed were analysed by nonparametric methods. Kruskall-Wallis test was performed to comparison of parameters between groups.

\section{RESULTS}

The mean age, sex and breeds of healthy dogs and dogs infected with vector borne diseases was summarized in Table 1. Classification of groups and related data were shown in Table 2, deemed there was no borrelliosis infection prevalent.

Statistically significant decreased PLT count was found in dogs mono infection with ehrlichiosis compared to healthy dogs $(P<0.001)$. Changes in mean $\mathrm{PT}$ value in the studied animals did not show statistically significant differences among the groups $(P>0.05)$. APTT values in the ehrlichiosis mono infection group were significantly higher than that of the healthy control $(P<0.001)$. A significant increase in FIB levels were detected for ehrlichiosis mono infection and ehrlichiosis - leishmaniosis co infection versus healthy control $(P<0.001)$. Plasma D-dimer concentrations were found to be higher in all groups infected with vector borne diseases compared to healthy group $(P<0.001)$ and the differences between infected groups were not statistically significant.

Table 1. Demographic characteristics of the healthy and dogs naturally infected with one or more vector-borne diseases (ehrlichiosis, anaplasmosis, leishmaniosis, dirofilariasis).

\begin{tabular}{cccc}
\hline & & Healthy $(\mathrm{n}=10)$ & Infected with vector-borne diseases $(\mathrm{n}=36)$ \\
\hline & Age (years) & $6.4 \pm 2.1$ & $4.6 \pm 3.9$ \\
\hline \multicolumn{2}{c}{ Sex (male/female) } & $4 / 6$ & $17 / 19$ \\
Breed & Cross breed & & 16 \\
$(\mathrm{n})$ & Labrador retreiver & 4 & 9 \\
& Anatolian shepherd dog & 2 & 6 \\
& German shepherd & 1 & 2 \\
\hline
\end{tabular}

Table 2. Platelet (PLT) count, prothrombin time (PT), activated partial thromboplastin time (APTT), fibrinogen (FIB) and D-dimer levels in healthy and dogs naturally infected with one or more vector-borne diseases.

\begin{tabular}{cccccc}
\hline Group & PLT $\left(\times 10^{9} / \mathrm{L}\right)$ & PT $(\mathrm{s})$ & APTT $(\mathrm{s})$ & FIB $(\mathrm{mg} / \mathrm{dL})$ & D-dimer $(\mathrm{mg} / \mathrm{L})$ \\
\hline Healthy $(\mathrm{n}=10)$ & $355 \pm 102^{\mathrm{a}}$ & $9.1 \pm 0.7$ & $12.7 \pm 1.1^{\mathrm{a}}$ & $163 \pm 57^{\mathrm{a}}$ & $0.09 \pm 0.0^{\mathrm{a}}$ \\
\hline Ehrlichiosis $(\mathrm{n}=12)$ & $104 \pm 146^{\mathrm{b}}$ & $9.1 \pm 2.0$ & $23.1 \pm 9.2^{\mathrm{b}}$ & $396 \pm 182^{\mathrm{b}}$ & $2.7 \pm 3.1^{\mathrm{b}}$ \\
\hline $\begin{array}{c}\text { Ehrlichiosis } \\
+\end{array}$ & $199 \pm 181^{\mathrm{ab}}$ & $8.5 \pm 1.3$ & $15.3 \pm 3.3^{\mathrm{ab}}$ & $306 \pm 134^{\mathrm{ab}}$ & $1.5 \pm 1.5^{\mathrm{b}}$ \\
& & & & $1.8 \pm 1.1^{\mathrm{b}}$ \\
Anaplasmosis $(\mathrm{n}=7)$ & & & & \\
\hline Leishmaniosis $(\mathrm{n}=6)$ & $457 \pm 206^{\mathrm{a}}$ & $13.0 \pm 10.8$ & $16.4 \pm 5.2^{\mathrm{ab}}$ & $316 \pm 221^{\mathrm{ab}}$ & $0.9 \pm 0.4^{\mathrm{b}}$ \\
\hline $\begin{array}{c}\text { Ehrlichiosis } \\
+\end{array}$ & $162 \pm 136^{\mathrm{ab}}$ & $9.5 \pm 0.97$ & $15.5 \pm 3.3^{\mathrm{ab}}$ & $343 \pm 164^{\mathrm{b}}$ & \\
\hline Leishmaniosis $(\mathrm{n}=6)$ & & & & $2.4 \pm 2.8^{\mathrm{b}}$ \\
\hline Dirofilariasis $(\mathrm{n}=5)$ & $470 \pm 432^{\mathrm{a}}$ & $12.4 \pm 9.7$ & $17.8 \pm 10.5^{\mathrm{ab}}$ & $208 \pm 113^{\mathrm{ab}}$ & 0.001 \\
\hline$P$ & 0.001 & 0.694 & 0.004 & 0.001 & \\
\hline
\end{tabular}

Statistical differences between groups were shown with different letters in the same column. 


\section{DISCUSSION}

Vector-borne diseases are among the principal concern to world public health, both in humans and animals. In veterinary medicine their impact is reflected by economic loss in livestock animals at major scale and home economies, not only due to health problems, but also due to control and treatments $[14,18]$. In dogs, the main health associated problems with vectors such as ticks, phlebotomine sand flies, and mosquitoes are transmission of diseases such as ehrlichiosis, anaplasmosis, babesiosis, hepatozoonosis, leishmaniasis and dirofilariasis [23,25]. The vector-borne diseases cause severe damage to various organs in the host. In the veterinary practice, fever, lethargy, anorexia, pale mucous membranes, lymphadenomegaly, splenomegaly, clotting disorders are reported as major clinical signs in dogs with vector-borne diseases $[6,8]$. The clinical signs associated with ehrlichiosis, anaplasmosis, leishmaniasis and dirofilariasis described in the dogs in this study were consistent with signs previously reported in the literature [25]. Prior to start of the study the present investigators thought that all selected vector borne diseases are prevalent in this region, whereas Lyme disease (borrelliosis) was neither evident nor prevalent [36].

There is little information on the evaluation of coagulation disorders in dogs naturally infected by one or more vector-borne pathogens. In most instances, published data describing coagulation alterations in dogs infected by one or more vector-borne pathogens derive from case reports, cross-sectional studies, or naturally infection studies involving single pathogens $[7,10,29,34]$. Bleeding disorders such as epistaxis, haematuria and haemorrhagic diarrhoea has been reported in dogs with vector-borne diseases [2,6]. These disorders represent the main cause of death in dogs, especially with ehrlichiosis. Thrombocytopenia usually presents with bleeding [6]. Thrombocytopenia is the most common manifestation of vector-borne diseases [8]. Low PLT numbers contribute significantly towards the morbidity and mortality of infection. Assessment of the PLT count in the diagnosis of primary haemostasis is an important step [17]. In the present study, thrombocytopenia was observed in dogs mono infected with ehrlichiosis compared to healthy dogs. This finding is in agreement with those reported in dogs with ehrlichiosis $[13,16]$. Bleeding disorder was seen only in a single dog with thrombocytopenia in the present study. Bleeding tendency could be related to this disorder as with ehrlichiosis. The pathogenic mechanism of PLT disorders in ehrlichiosis is not clear. Thrombocytopenia in ehrlichiosis has been associated with increased PLT consumption due to immune-mediated destruction and sequestration in the spleen, decreased production of PLT, or combination of these mechanisms $[4,17,24]$.

The FIB in the glycoprotein structure, designated factor I, is synthesized by the liver and converted to fibrin by thrombin during blood coagulation [12]. Plasma FIB is one of the most important factors in the coagulation cascade and its concentration can be elevated in other clinical conditions such as infections, haemodynamic impairment, cardiac, lung and aortic diseases and malignancies, as an acute-phase reactant [15]. The blood FIB concentration may increase in acute and chronic conditions as an acutephase reactant in canine ehrlichiosis. There are few studies on the relationship between FIB concentration and vector-borne diseases in dogs $[6,27]$. Edirimanne et al. [9] reported that FIB levels were significantly higher in dogs with ehrlichiosis. In another study, Ciaramella et al. [6] showed that FIB concentrations were no significantly higher in dogs with markedly symptomatic leishmaniosis. In the present study, a significant difference between dogs with ehrlichiosis mono infection and ehrlichiosis - leishmaniosis co infection versus healthy controls group was observed. The increases in FIB concentrations are likely due to increased rate of synthesis probably stimulated by cytokines, growth factors, hormones, and other cellular effectors [3].

PT and APTT are commonly used coagulation biomarkers for interpretation of dogs with bleeding tendencies. In a prior study, the APTT values were significantly elevated in dogs with ehrlichiosis [31]. Another study comprising dogs, prolonged APTT was found during leishmaniosis mono infection [6], and ehrlichiosis - leishmaniosis co infection [7], in particular when accompanied by liver dysfunctions lowering the synthesis of on ore more coagulation factors. In current study, a significant difference between dogs with ehrlichiosis and healthy control, was observed in APTT values, however, differences in PT values compared to healthy dogs were insignificant. No statistical difference in PT values might be related to the lower sensitivity of the commercial PT assays 
[19]. Prolonged APTT and normal PT values could be due to deficiency of coagulation factors VIII, IX and XI [19,33].

D-dimer is a degradation product of crosslinked fibrin that may be increased with clot formation and fibrinolysis [21,22]. In dogs, D-dimer concentrations can be elevated due to disseminated intravascular coagulopathy (DIC), infections, metabolic disorders, neoplasia and post-surgically $[11,30,37]$. This parameter is also one of few indicators of a prothrombotic state that leads to DIC [32]. In the present study, a significant increase in D-dimer concentration was observed in all dogs with vector-borne diseases. This finding points out the activation of the fibrinolysis system in consequence of thrombophilia [20]. An increase in the value of D-dimer was determined in one of the two dogs infected with Anaplasma phagocytophilum [10]. In another study, Carretón et al. [5] reported that D-dimer concentrations were significantly higher in dogs with dirofilariasis.

\section{CONCLUSIONS}

In conclusion, elevations presented in coagulation biomarkers such as APTT, FIB and D-dimer in the present study were interpreted as with the effects of vector-borne diseases. It may be briefly suggested that D-dimer levels as a marker of pro-coagulatory activity, as well as fibrinolysis, indicates the highly active and excessive coagulation, and all through are risk factors for thromboembolic disorders. Therefore, these findings should be considered in the diagnosis, treatment and prognosis of the vector-borne diseases in dogs.

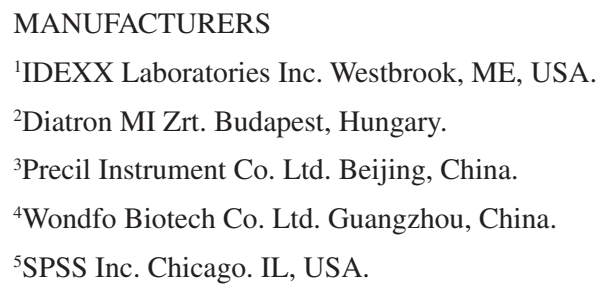

Acknowledgements. This study was summarized partially from a national Project and was funded by Adnan Menderes University Research Projects Funding Unit with project number VTF-15049. The researchers who put pen, their heart and great effort to this study were involved.

Ethical approval. The study protocol was approved by the institutional laboratory animals ethics committee (ADU-HADYEK $64583101 / 2014 / 163$ ). Informed written consent was obtained from all of the owners/animal care takers prior to enrolment.

Declaration of interest. The authors report no conflicts of interest. The authors alone are responsible for the content and writing of paper.

\section{REFERENCES}

1 Baneth G., Harrus S., Ohnona F.S. \& Schlesinger Y. 2009. Longitudinal quantification of Ehrlichia canis in experimental infection with comparison to natural infection. Veterinary Microbiology. 136: 321-325.

2 Bhadesiya C.M. \& Raval S.K. 2015. Hematobiochemical changes in ehrlichiosis in dogs of Anand region, Gujarat. Veterinary World. 8(6): 713-717.

3 Blomback B. 1996. Fibrinogen and fibrin-proteins withcomplex roles in hemostasis and thrombosis. Thrombosis Research. 83: 1-75.

4 Bulla C., Kiomi Takahira R., Pessoa Araújo J., Aparecida Trinca L., Souza Lopes R. \& Wiedmeyer C.E. 2004. The relationship between the degree of thrombocytopenia and infection with Ehrlichia canis in an endemic area. Veterinary Research. 35(1): 141-146.

5 Carretón E., Corbera J.A., Juste M.C., Morchón R., Simón F. \& Montoya-Alonso J.A. 2011. Dirofilaria immitis infection in dogs: cardiopulmonary biomarker levels. Veterinary Parasitology. 176: 313-316.

6 Ciaramella P., Pelagalli A., Cortese L., Pero M.E., Corona M., Lombardi P., Avallone L. \& Persechino A. 2005. Altered platelet aggregation and coagulation disorders related to clinical findings in 30 dogs naturally infected by Leishmania infantum. Veterinary Journal. 169: 465-467.

7 Cortese L., Pelagalli A., Piantedosi D., Mastellone V., Manco A., Lombardi P., Ciaramella P. \& Avallone L. 2006. Platelet aggregation and haemostatic response in dogs naturally co-infected by Leishmania infantum and Ehrlichia canis. Journal of Veterinary Medicine. 53: 546-548.

8 Caprariis D., Dantas-Torres F., Capelli G., Mencke N., Stanneck D., Breitschwerdt E.B. \& Otranto D. 2011. Evolution of clinical, haematological and biochemical findings in young dogs naturally infected by vector-borne pathogens. Veterinary Microbiology. 149(1-2): 206-212.

9 Edirimanne E.R.K.V., Ariyarathna H.S., Beijerink N.J. \& de Silva D.D.N. 2014. Latest clinical manifestations of canine ehrlichiosis: “The Pup Killer” - A Preliminary Communication. Wayamba Journal of Animal Science. 1397905837: 880-892. 
10 Elhamiani Khatat S., Defauw P., Marynissen S., Van de Maele I., van Dongen A. \& Daminet S. 2015. Exposure to Anaplasma phagocytophilum in two dogs in Belgium. Vlaams Diergeneeskundig Tijdschrift. 84(1): 39-46.

11 Epstein S.E., Hopper K., Mellema M.S. \& Johnson L.R. 2013. Diagnostic utility of D-dimer concentrations in dogs with pulmonary embolism. Journal of Veterinary Internal Medicine. 27: 1646-1649.

12 Everse S.J., Spraggon G., Veerapandian L., Riley M. \& Doolittle R.F. 1998. Crystal structure of fragment double-D from human fibrin with two different bound ligands. Biochemistry. 37(24): 8637-8642.

13 Gaunt S.D., Baker D.C. \& Babin S.S. 1990. Platelet aggregation studies in dogs with acute Ehrlichia platys infection. American Journal of Veterinary Research. 51(2): 290-293.

14 Grisi L., Leite R.C., Martins J.R., Barros A.T., Andreotti R. \& Cançado P.H. 2014. Reassessment of the potential economic impact of cattle parasites in Brazil. Revista Brasileira de Parasitologia Veterinária. 23: 150-156.

15 Hajsadeghi S., Kerman S.R., Khojandi M., Vaferi H., Ramezani R., Jourshari N.M., Mousavi S.A. \& Pouraliakbar H. 2012. Accuracy of D-dimer:fibrinogen ratio to diagnose pulmonary thromboembolism in patients admitted to intensive care units. Cardiovascular Journal of Africa. 23(8): 446-456.

16 Harrus S., Waner T., Eldor A., Zwang E. \& Bark H. 1996. Platelet dysfunction associated with experimental acute canine ehrlichiosis. Veterinary Record. 139(12): 290-293.

17 Herring J. \& Mc Michael M. 2012. Diagnostic approach to small animal bleeding disorders. Topics in Companion Animal Medicine. 27: 73-80.

18 Irwin PJ. 2014. It shouldn't happen to a $\operatorname{dog} . .$. or a veterinarian: clinical paradigms for canine vector-borne diseases. Trends in Parasitology. 30: 104-112.

19 Jimenez C. \& Fernandez F. 2000. Inflammation, kinins, and complement. System interaction with haemostasis. In: B.F. Feldman, J.G. Zinkl \& N.C. Jain (Eds). Schalm's Veterinary Hematology. 5th edn. Philadelphia: Lippincott Williams and Wilkins, pp.549-551.

20 Kang M.H., Heo R.Y. \& Park H.M. 2016. Evaluation of D-dimer concentrations in clinically ill dogs with high risk of thromboembolic disease. Pakistan Veterinary Journal. 36(2): 219-223.

21 Kebapcilar A.G., Kulaksizoglu M., Ipekci S.H., Korkmaz H., Kebapcilar L., Akyurek F., Taner C.E. \& Gonen M.S. 2013. Relationship between mean platelet volume and low-grade systemic coagulation with vitamin D deficiency in primary ovarian insufficiency. Archives of gynecology and obstetrics. 288(1): 207-212.

22 Koenig W. 1998. Haemostatic risk factors for cardiovascular diseases. European Heart Journal. 19: 39-43.

23 Kubo S., Tateno M., Ichikawa Y. \& Endo Y. 2015. A molecular epidemiological survey of Babesia, Hepatozoon, Ehrlichia and Anaplasma infections of dogs in Japan. Journal of Veterinary Medicine and Science. 77: 1275-1279.

24 Lakkawar W.A., Nair G.M., Varshney C.K., Sreekrishnan R. \& Rao V.N. 2003. Pathology of canıne monocytic ehrlichiosis in a German shepherd dog. Slovenian Veterinary Research. 40 (2): 119-128.

25 Otranto D. \& Dantas-Torres F. 2010. Canine and feline vector-borne diseases in Italy: current situation and perspectives. Parasite \& Vectors. 3: 2.

26 Otranto D., Dantas-Torres F. \& Breitschwerdt E.B. 2009. Managing canine vector-borne diseases of zoonotic concern: part one. Trends in Parasitology. 25: 157-163.

27 Ruiz de Gopegui R., Peñalba B., Goicoa A., Espada Y., Fidalgo L.E. \& Espino L. 2007. Clinico-pathological findings and coagulation disorders in 45 cases of canine babesiosis in Spain. Veterinary Journal. 174(1): 129-132.

28 Santamaria A., Calzada J.E., Saldaña A., Yabsley M.J. \& Gottdenker N.L. 2014. Molecular diagnosis and species identification of Ehrlichia and Anaplasma infections in dogs from Panama, Central America. Vector Borne Zoonotic Diseases. 14: 368-370.

29 Schetters T.P., Kleuskens J.A., Van De Crommert J., De Leeuw P.W., Finizio A.L. \& Gorenflot. A. 2009. Systemic inflammatory responses in dogs experimentally infected with Babesia canis; a haematological study. Veterinary Parasitology. 162: 7-15.

30 Scott-Moncrieff J.C., Treadwell N.G., McCullough S.M. \& Brooks M.B. 2001. Hemostatic abnormalities in dogs with primary immune-mediated hemolytic anemia. Journal of American Animal Hospital Association. 37: 220-227.

31 Shipov A., Klement E., Reuveni-Tager L., Waner T. \& Harrus S. 2008. Prognostic indicators for canine monocytic ehrlichiosis. Veterinary Parasitology. 153(1-2): 131-138.

32 Stokol T., Brooks M.B., Erb H.N. \& Mauldin G.E. 2000. D-dimer concentrations in healthy dogs and dogs with disseminated intravascular coagulation. American Journal of Veterinary Research. 61: 393-398. 
33 Sumathi D., Selvaraj P., Nambi A.P., Prathaban S. \& Enbavelan P.A. 2012. Assessment of prothrombin and activated partial thromboplastin time in dogs. Tamilnadu Journal of Veterinary \& Animal Sciences. 8(4): 238-240.

34 Torres Mde M., Almeida Ado B., Paula D.A., Mendonça A.J., Nakazato L., Pescador C.A. \& Sousa V.R. 2016. Hemostatic assessment of dogs associated with hepatic parasite load of Leishmania infantum chagasi. Revista Brasileira de Parasitologia Veterinária. 25(2): 244-247.

35 Tripodi A., Branchi A. \& Chantarangkul V. 2011. Hypercoagulability in patients with type 2 diabetes mellitus detected by a thrombin generation assay. Journal of Thrombosis and Thrombolysis. 31: 165-172.

36 Ural K., Gultekin M., Atasoy A. \& Ulutas B. 2014. Spatial distribution of vector borne disease agents in dogs in Aegean region, Turkey. Revista MVZ Córdoba. 19(2): 4086-4098.

37 Zoia A., Augusto M., Drigo M. \& Caldin M. 2012. Evaluation of hemostatic and fibrinolytic markers in dogs with ascites attributable to right-sided congestive heart failure. Journal of American Veterinary Medical Association. 241: $1336-1343$. 